\title{
Complications in pregnancies achieved by assisted reproduction
}

José M. Madrazo-Cabo, Grecia A. León-Durán, Adriana Oliveros-Montiel, José A. Ledesma-Montes, Iván D. Morales-González and VirginiaSedeño-Monge*

Universidad Popular Autónoma del Estado de Puebla, Health Sciences Deanship, Faculty of Medicine, Puebla, Mexico

\begin{abstract}
Introduction: Pregnancies resulting from assisted reproductive technologies (ART) have been documented to have a higher risk of adverse effects. Objective: To provide evidence on obstetric and perinatal complications associated with conceptions by ART versus spontaneous pregnancies. Method: Comprehensive review of original articles published between 2010 and 2018 addressing the more common obstetric and perinatal complications in pregnancies resulting from in vitro fertilization (IVF) and intracytoplasmic sperm injection (ICSI), in comparison with spontaneous conceptions. Results: Thirty-seven original articles, which reported on 26 cohort studies and 11 case-control trials, were included. IVF and ICSI conceptions were associated with a larger number of obstetric and perinatal complications such as low birth weight, prematurity, low weight for gestational age, admission to the neonatal intensive care unit, congenital malformations, $C$-sectionand premature rupture of membranes, among others. Conclusions: Pregnancies by ART are associated with an increased risk of obstetric and perinatal complications in comparison with spontaneous conceptions. Further research is needed to determine which aspects result in higher risk.
\end{abstract}

KEY WORDS: Assisted human reproduction. In vitro fertilization. Intracytoplasmic sperm injection.

\section{Complicaciones en embarazos logrados por reproducción asistida}

\section{Resumen}

Introducción: Se ha documentado que los embarazos por técnicas de reproducción asistida (TRA) presentan mayor riesgo de efectos adversos. Objetivo: Proporcionar evidencia actualizada de las complicaciones obstétricas y perinatales asociadas con concepciones mediante TRA versus embarazos espontáneos. Método: Revisión de artículos originales publicados entre 2010 y 2018, que abordan complicaciones obstétricas y perinatales de mayor frecuencia en embarazos por fertilización in vitro (FIV) e inyección intracitoplasmática de espermatozoides (ICSI) comparados con concepciones espontáneas. Resultados: Se incluyeron 37 artículos originales, 26 de cohorte y 11 de casos y controles. Las concepciones por FIV e ICSI se asociaron con más complicaciones obstétricas y perinatales como bajo peso al nacimiento, prematuridad, menor peso para la edad gestacional, ingreso a la unidad de cuidados intensivos neonatales, malformaciones congénitas, cesárea, ruptura prematura de membranas, entre otras. Conclusiones: Las concepciones por TRA se asocian con mayor riesgo de complicaciones obstétricas y perinatales en comparación con las espontáneas. Es necesario realizar estudios adicionales que determinen qué aspectos derivan en mayor riesgo.

PALABRAS CLAVE: Reproducción humana asistida. Fertilización in vitro. Inyección intracitoplasmática de espermatozoides.

\footnotetext{
Correspondence:

*Virginia Sedeño-Monge

E-mail: virginia.sedeno@upaep.mx

Gac Med Mex. 2020;156:156-163

Contents available at PubMed

www.gacetamedicademexico.com

0016-3813/@ 2020 Academia Nacional de Medicina de México, A.C.. Published by Permanyer. This is an open access article under the CC BY-NC-ND license (http://creativecommons.org/licenses/by-nc-nd/4.0/).
} 


\section{Introduction}

Each year, more than 200,000 babies are born worldwide as a result of the use of assisted reproductive technologies (ART):1,2 to date, approximately 7 million children conceived through this modality have been born.

In in vitro fertilization (IVF), the ovaries are stimulated with medications and are subsequently aspirated from the ovarian follicles and fertilized in vitro; the retrieved oocytes are mixed with sperm in a culture medium, and then are transferred to the uterine cavity. ${ }^{3}$

As part of an IVF cycle, a single sperm is injected into the cytoplasm of a mature oocyte (intracytoplasmic sperm injection, ICSI), which is an efficacious method to help fertilization in men with suboptimal semen parameters or with low fertilization rates.

As experience was accumulated, success rates increased and indications for these procedures were expanded, with little formal evaluation of their effects on maternal and fetal well-being. ${ }^{4}$ Several studies have indicated that ART can generate a higher risk of maternal and perinatal complications in comparison with spontaneous conceptions. ${ }^{5}$

\section{Method}

A review of original articles published in indexed journals such as PubMed, Medline, CONRICyT, Medigraphic and SciELO between 2010 and 2018 was carried out. The keywords used were "assisted reproductive techniques", "in vitro fertilization", "IVF", "intracytoplasmic sperm injection", "ICSI", "perinatal outcomes" and "obstetric outcomes". Articles comparing obstetric and perinatal complications between IVF and ICSI pregnancies and spontaneous conceptions, both cohort and case-control studies, were included. Meta-analyses, systematic reviews and articles not having spontaneous pregnancies as a control group were excluded.

\section{Results}

Thirty-seven trials were included, out of which 26 were cohort and 11 case-control studies. Data on each study are shown in Table 1.

The results are described according to the type of complication and to the type of articles in which the relative risk of finding these complications was statistically higher $(p<0.05)$ in ART-induced pregnancies versus spontaneous conceptions.

\section{Complications}

- Prematurity: complication reported in 26 articles (70\% of total), in 67,186 pregnancies resulting from IVF-ICSI; gestational age recorded at birth ranged from 32 to 37 weeks. In 18 articles, the frequency of prematurity was significantly higher with ART in comparison with spontaneous pregnancies..$^{6-23}$

- Low birth weight: this complication was reported in 21 articles (59,140 ART-related births) (57\% of total). Less than $2,500 \mathrm{~g}$ was considered low birth weight, and less than $1,500 \mathrm{~g}$ was regarded as very low weight; in five articles, significant values were reported for very low weight ${ }^{6,12,17,18,22}$ and in 14 for low weight in pregnancies resulting from ART, in comparison with spontaneous pregnancies. $6,8,9,12,13,16-19,22,24-27$

- Perinatal mortality: this complication is defined as intrauterine death of a fetus weighing less than $500 \mathrm{~g}$ or having less than 20 weeks of gestational development and that of neonates within the first seven days of life. ${ }^{9,18,28,29}$ Out of 18 articles (49\% of total) that included 591 pregnancies resulting from IVF-ICSI and in which perinatal mortality was reported, seven recorded significant mortality values in ART pregnancies in comparison with spontaneous pregnancies. ${ }^{12,17,18,22,23,30,31}$

- Small for gestational age (SGA): it is defined as a child with birth weight below the $75^{\text {th }}$ percentile ${ }^{28}$ or weighing less than 2 standard deviations than the population for that gestational age. ${ }^{17}$ In 13 articles (35\% of total) that included 10,631 IVF-ICSI, this complication was reported; in four, the result was significant. ${ }^{12,17,24,32}$

- Admission to the neonatal intensive care unit (NICU): this complication was reported in eight articles that included 604 IVF-ICSI; five studies showed significant difference in pregnancies obtained by means of ART.9,18,19,22,23

- Congenital malformations: Twenty articles (54\% of total), which included 7288 IVF-ICSI procedures, report congenital malformations; cardiovascular malformations were the most common in 10 articles. In comparison with spontaneous conceptions, an increased trend to find any congenital defect was reported in pregnancies 
Gaceta Médica de México. 2020;156

Table 1. Characteristics of studies published in indexed journals between 2010 and 2018 addressing obstetric and perinatal complications between pregnancies resulting from IVF and ICSI and spontaneous conceptions

\begin{tabular}{|c|c|c|c|c|}
\hline Study & Country, period & Design & Population & Reported complications \\
\hline $\begin{array}{l}\text { Bassioun et al., } \\
2014^{23}\end{array}$ & $\begin{array}{l}\text { Egypt, } \\
\text { January } \\
2010-\text { December } \\
2012\end{array}$ & Case-control & $\begin{array}{l}\text { Cases: } 739 \text { ICSI } \\
\text { Controls: } 843 \\
\text { spontaneous } \\
\text { pregnancies }\end{array}$ & $\begin{array}{l}\text { The ICSI group was associated with a higher incidence } \\
\text { of twins } 12.7 \%(p<0.001) \text {, premature delivery } 3.8 \% \\
(p=0.022) \text {, PROM } 4.6 \%(p=0.001) \text {, C-section } 74.1 \% \\
(p<0.001) \text { and neonatal deaths } 10.4 \%(p<0.001)\end{array}$ \\
\hline $\begin{array}{l}\text { Boulet et al., } \\
2016^{35}\end{array}$ & $\begin{array}{l}\text { United States, } \\
2000-2010\end{array}$ & $\begin{array}{l}\text { Retrospective } \\
\text { cohort }\end{array}$ & $\begin{array}{l}\text { Exposed cohort: } 64,861 \\
\text { IVF-ICSI } \\
\text { Non-exposed cohort: } \\
4,553,215 \text { spontaneous } \\
\text { pregnancies }\end{array}$ & $\begin{array}{l}\text { The prevalence of one or more non-chromosomal } \\
\text { defects was 58.59/10,000 children conceived with ART } \\
(n=389) \text { versus } 47.50 / 10,000 \text { in children conceived } \\
\text { without ART }(n=22,036) ; \mathrm{aOR}=1.28 \\
95 \% \mathrm{Cl}=1.15-1.42\end{array}$ \\
\hline $\begin{array}{l}\text { Caserta et al., } \\
201^{49}\end{array}$ & $\begin{array}{l}\text { Italy, } \\
\text { January 2007-June } \\
2011\end{array}$ & Case-control & $\begin{array}{l}\text { Cases: } 138 \text { IVF-ICSI } \\
\text { Controls: } 207 \\
\text { spontaneous } \\
\text { pregnancies }\end{array}$ & $\begin{array}{l}\text { Gestational age and birth weight were lower in the } \\
\text { ART group. The frequency of diabetes and placental } \\
\text { abruption was higher for ART in comparison with } \\
\text { spontaneous pregnancies. Pregnancies by ART had a } \\
\text { higher incidence of placental abruption (OR }=7.45 \text {, } \\
95 \% \mathrm{Cl}=2.05-26.98) \text { and patent ductus arteriosus } \\
(\mathrm{OR}=3.39,95 \% \mathrm{Cl}=1.01-11.46) \text {. }\end{array}$ \\
\hline $\begin{array}{l}\text { Farhi et al., } \\
2013^{20}\end{array}$ & $\begin{array}{l}\text { Israel, June 2006- } \\
\text { December } 2008\end{array}$ & $\begin{array}{l}\text { Prospective } \\
\text { cohort }\end{array}$ & $\begin{array}{l}\text { Exposed cohort: } 561 \text { ART } \\
\text { (223 IVF and } 338 \text { ICSI) } \\
\text { Non-exposed cohort: } 600 \\
\text { spontaneous } \\
\text { pregnancies. }\end{array}$ & $\begin{array}{l}\text { Pregnancies through IVF were associated with } \\
\text { prematurity }(\mathrm{OR}=2.36,95 \% \\
\mathrm{Cl}=1.28-4.37) \text { and low birth weight } \\
(\mathrm{OR}=1.89,95 \% \mathrm{Cl}=1.03-3.46)\end{array}$ \\
\hline $\begin{array}{l}\text { Farhi et al., } \\
2013^{21}\end{array}$ & $\begin{array}{l}\text { Israel, } \\
\text { 1997-2004 }\end{array}$ & $\begin{array}{l}\text { Retrospective } \\
\text { cohort }\end{array}$ & $\begin{array}{l}\text { Exposed cohort: } 9042 \\
\text { IVF-ICSI Non-exposed } \\
\text { cohort: } 213,288 \\
\text { spontaneous } \\
\text { pregnancies }\end{array}$ & $\begin{array}{l}\text { Higher risk of CM with ART ( } 2.4 \% \text { versus } 1.9 \% \text {, } \\
\text { aOR }=1.45,95 \% \mathrm{Cl}=1.26-1.68) \text {. In single NBs, the risk } \\
\text { for nervous system disorders was increased (OR }=2.13 \text {, } \\
95 \% \mathrm{Cl}=1.04-4.37) \text {, as well as for circulatory system } \\
(\mathrm{OR}=1.47,95 \% \mathrm{Cl}=1.09-1.98) \text {, digestive system } \\
(\mathrm{OR}=3.10,95 \% \mathrm{Cl}=1.48-6.48) \text { and genital apparatus } \\
\text { disorders }(\mathrm{OR}=1.60,95 \% \mathrm{Cl}=1.0-2.52) \text {, in the group } \\
\text { of ART pregnancies versus spontaneous pregnancies }\end{array}$ \\
\hline $\begin{array}{l}\text { Harlev et al., } \\
2018^{15}\end{array}$ & $\begin{array}{l}\text { Israel, January } 1991 \\
\text {-December } 2013\end{array}$ & Case-control & $\begin{array}{l}\text { Cases: } 229 \text { IVF } \\
\text { Controls: } 7929 \\
\text { spontaneous } \\
\text { pregnancies }\end{array}$ & $\begin{array}{l}\text { Preterm delivery }(p<0.001) \text {, growth restriction } \\
(p<0.001) \text { and } C \text {-section }(p<0.001) \text { had high } \\
\text { prevalence in pregnancies resulting from ART (IVF) }\end{array}$ \\
\hline $\begin{array}{l}\text { Healy et al., } \\
2010^{42}\end{array}$ & $\begin{array}{l}\text { Australia, } \\
\text { 1991-2004 }\end{array}$ & $\begin{array}{l}\text { Retrospective } \\
\text { cohort }\end{array}$ & $\begin{array}{l}\text { Exposed cohort: } 6730 \\
\text { IVF-ICSI. Non-exposed } \\
\text { cohort: } 24,619 \\
\text { spontaneous pregnancies }\end{array}$ & $\begin{array}{l}\text { The IVF-ICSI group had a higher incidence of } \\
\text { postpartum hemorrhage }(6.7 \% \text { versus } 3.6 \% \text {, } \\
\text { aOR }=2.0,95 \% \mathrm{Cl}=1.8-2.3)\end{array}$ \\
\hline $\begin{array}{l}\text { Henningsen } \\
\text { et al., 201116 }\end{array}$ & $\begin{array}{l}\text { Denmark, } \\
\text { 1994-2008 }\end{array}$ & $\begin{array}{l}\text { Retrospective } \\
\text { cohort }\end{array}$ & $\begin{array}{l}\text { Exposed cohort: } 3881 \\
\text { IVF-ICSI Non-exposed } \\
\text { cohort: } 7758 \text { spontaneous } \\
\text { pregnancies }\end{array}$ & $\begin{array}{l}\text { Higher risk of } \mathrm{LBW}(\mathrm{OR}=1.4,95 \% \mathrm{Cl}=1.1-1.7) \text { and } \\
\text { prematurity }(\mathrm{OR}=1.3,95 \% \mathrm{CI}=1.1-1.6) \text { with } \mathrm{IVF}-\mathrm{ICSI} \\
\text { in comparison with spontaneous pregnancies }\end{array}$ \\
\hline $\begin{array}{l}\text { Jackson et al., } \\
2015^{38}\end{array}$ & $\begin{array}{l}\text { United States, } \\
\text { January } \\
\text { 2000-October } 2010\end{array}$ & $\begin{array}{l}\text { Retrospective } \\
\text { cohort }\end{array}$ & $\begin{array}{l}\text { Exposed cohort: } 185 \text { IVF } \\
\text { Non-exposed cohort: } 193 \\
\text { spontaneous } \\
\text { pregnancies }\end{array}$ & $\begin{array}{l}\text { Increased risk of placenta accreta ( } 2.7 \% \text { versus } 0 \% \text { ) } \\
\text { and elective C-section ( } 75.1 \% \text { versus } 49.7 \% \text { ) in women } \\
\text { who conceived by means of ART in comparison with } \\
\text { those with spontaneous pregnancy }\end{array}$ \\
\hline $\begin{array}{l}\text { Källen et al., } \\
2010^{6}\end{array}$ & $\begin{array}{l}\text { Sweden, } \\
1982-2007\end{array}$ & $\begin{array}{l}\text { Retrospective } \\
\text { cohort }\end{array}$ & $\begin{array}{l}\text { Exposed cohort: } 1545 \\
\text { twins by IVF. } \\
\text { Non-exposed cohort: } \\
8675 \text { spontaneous twins }\end{array}$ & $\begin{array}{l}\text { Higher risk of premature birth }<32 \text { WOG }(\mathrm{aOR}=1.52 \text {, } \\
95 \% \mathrm{Cl}=1.18-1.97), \mathrm{LBW}<500 \mathrm{~g}(\mathrm{OR}=1.54,95 \% \\
\mathrm{Cl}=1.25-1.89) \text { in the IVF group }\end{array}$ \\
\hline $\begin{array}{l}\text { Källén et al., } \\
2010^{37}\end{array}$ & $\begin{array}{l}\text { Sweden, } \\
1982-2007\end{array}$ & Case-control & $\begin{array}{l}\text { Cases: } 15,570 \text { IVF } \\
\text { Controls: } 689,157 \\
\text { spontaneous pregnancies }\end{array}$ & $\begin{array}{l}\text { Increased risk of congenital malformations }(\mathrm{OR}=1.23 \text {, } \\
95 \% \mathrm{Cl}=1.14-1.32) \text { associated with IVF }\end{array}$ \\
\hline $\begin{array}{l}\text { Kosteria et al., } \\
2017^{39}\end{array}$ & Greece & Case-control & $\begin{array}{l}\text { Cases: } 42 \text { ICSI. } \\
\text { Controls: } 42 \text { spontaneous } \\
\text { pregnancies }\end{array}$ & $\begin{array}{l}\text { The ICSI group had a shorter gestation, more C-sections } \\
(p=0.003) \text {, lower birth weight and length }\end{array}$ \\
\hline
\end{tabular}


Table 1. Characteristics of studies published in indexed journals between 2010 and 2018 addressing obstetric and perinatal complications between pregnancies resulting from IVF and ICSI and spontaneous conceptions (Continued)

\begin{tabular}{|c|c|c|c|c|}
\hline Study & Country, period & Design & Population & Reported complications \\
\hline $\begin{array}{l}\text { Lerner-Geva } \\
\text { et al., } 2017^{27}\end{array}$ & $\begin{array}{l}\text { Israel, } \\
\text { 1994-2004 }\end{array}$ & $\begin{array}{l}\text { Historical } \\
\text { cohort }\end{array}$ & $\begin{array}{l}\text { Exposed cohort: } 9042 \text { IVF } \\
\text { Non-exposed cohort: } \\
211,763 \text { spontaneous } \\
\text { pregnancies }\end{array}$ & $\begin{array}{l}\text { Children conceived with ART had a significant risk for } \\
\text { specific cancer such as retinoblastoma }(\mathrm{OR}=6.18 \text {, } \\
95 \% \mathrm{Cl}=1.22-31.2) \text { and renal tumors ( } \mathrm{OR}=3.25,95 \% \\
\mathrm{Cl}=1.67-6.32)\end{array}$ \\
\hline $\begin{array}{l}\text { Messerschmidt } \\
\text { et al., } 2010^{32}\end{array}$ & $\begin{array}{l}\text { Austria, } \\
\text { 1999-2007 }\end{array}$ & $\begin{array}{l}\text { Retrospective } \\
\text { cohort }\end{array}$ & $\begin{array}{l}\text { Exposed cohort: } 195 \mathrm{IVF} \\
\text { Non-exposed cohort: } 1228 \\
\text { spontaneous pregnancies }\end{array}$ & $\begin{array}{l}\text { Preterm births were more common in multiple } \\
\text { pregnancies obtained by IVF }(81.8 \%) \text { unlike } \\
\text { spontaneous pregnancies }(57.2 \%) \text { and SGA }(p<0.01)\end{array}$ \\
\hline $\begin{array}{l}\text { Min Yang et al., } \\
2018^{31}\end{array}$ & $\begin{array}{l}\text { China, } \\
\text { 2006-2016 }\end{array}$ & $\begin{array}{l}\text { Retrospective } \\
\text { cohort }\end{array}$ & $\begin{array}{l}\text { Exposed cohort: } 2484 \text { IVF } \\
\text { Non-exposed cohort: } \\
109,559 \text { spontaneous } \\
\text { pregnancies }\end{array}$ & $\begin{array}{l}\text { ART conceptions had a higher risk for any type of birth } \\
\text { defect in comparison with spontaneous pregnancies } \\
\text { (aOR } 2.10,95 \% \mathrm{Cl}=1.63-2.69 \text { ) }\end{array}$ \\
\hline $\begin{array}{l}\text { Moini et al., } \\
2012^{18}\end{array}$ & $\begin{array}{l}\text { Iran, } \\
\text { January } \\
\text { 2008-October } 2010\end{array}$ & $\begin{array}{l}\text { Prospective } \\
\text { cohort }\end{array}$ & $\begin{array}{l}\text { Exposed cohort: } 420 \text { IVF } \\
\text { Non-exposed cohort: } 340 \\
\text { spontaneous } \\
\text { pregnancies }\end{array}$ & $\begin{array}{l}\text { Preterm birth rates }(\mathrm{OR}=5.2,95 \% \mathrm{Cl}=2.1-12.9), \mathrm{LBW} \\
(\mathrm{OR}=2.2,95 \% \mathrm{Cl}=1.0-3.9), \mathrm{NICU} \text { admission } \\
(\mathrm{OR}=2.0,95 \% \mathrm{Cl}=1.2-3.2) \text { and perinatal mortality } \\
(\mathrm{OR}=2.3,95 \% \mathrm{Cl}=1.2-4.0) \text { were significant for ART }\end{array}$ \\
\hline $\begin{array}{l}\text { Mozafari } \\
\text { Kermani et al., } \\
2018^{36}\end{array}$ & $\begin{array}{l}\text { Iran, } \\
\text { January } \\
\text { 2012-december } \\
2014\end{array}$ & $\begin{array}{l}\text { Historical } \\
\text { cohort }\end{array}$ & $\begin{array}{l}\text { Exposed cohort: } 168 \\
\text { IVF-ICSI. } \\
\text { Non-exposed cohort: } 652 \\
\text { spontaneous pregnancies }\end{array}$ & $\begin{array}{l}\text { Children conceived with ART had twice the risk of CM } \\
(p=0.046) \text { in comparison with those conceived by } \\
\text { spontaneous pregnancy }\end{array}$ \\
\hline $\begin{array}{l}\text { Opdahl et al., } \\
2015^{43}\end{array}$ & $\begin{array}{l}\text { Sweden, Denmark } \\
\text { and Norway, } \\
\text { 1988-2007 }\end{array}$ & $\begin{array}{l}\text { Retrospective } \\
\text { cohort }\end{array}$ & $\begin{array}{l}\text { Exposed cohort: } 58,006 \\
\text { IVF-ICSI } \\
\text { Non-exposed cohort: } \\
315,273 \text { spontaneous } \\
\text { pregnancies }\end{array}$ & $\begin{array}{l}\text { The risk of hypertensive disorders of pregnancy } \\
\text { was higher in the ART group ( } O R=1.16,95 \% \\
\mathrm{Cl}=1.10-1.21)\end{array}$ \\
\hline $\begin{array}{l}\text { Panagiotopoulou } \\
\text { et al., } 2016^{34}\end{array}$ & $\begin{array}{l}\text { Greece, } \\
1995-2012\end{array}$ & $\begin{array}{l}\text { Retrospective } \\
\text { cohort }\end{array}$ & $\begin{array}{l}\text { Exposed cohort: } 389 \\
\text { IVF-ICSI. } \\
\text { Non-exposed cohort: } 485 \\
\text { spontaneous pregnancies }\end{array}$ & $\begin{array}{l}\text { In the ART group, } 8.2 \% \text { had congenital cardiovascular } \\
\text { disease in comparison with } 4.3 \% \text { of those naturally } \\
\text { conceived }(\mathrm{OR}=1.90,95 \% \mathrm{Cl}=1.08-3.34, \mathrm{p}=0.024)\end{array}$ \\
\hline $\begin{array}{l}\text { Pelkonen et al., } \\
2014^{11}\end{array}$ & $\begin{array}{l}\text { Finland, } \\
\text { 1995-2006 }\end{array}$ & $\begin{array}{l}\text { Retrospective } \\
\text { cohort }\end{array}$ & $\begin{array}{l}\text { Exposed cohort: } 4772 \text { IVF. } \\
\text { Non-exposed cohort: } \\
31243 \text { spontaneous } \\
\text { pregnancies }\end{array}$ & $\begin{array}{l}\text { Children of ART pregnancies had a higher risk of major } \\
\mathrm{CM}(\mathrm{OR}=1.24,95 \% \mathrm{Cl}=1.05-1.47) \text { in comparison with } \\
\text { those spontaneously conceived }\end{array}$ \\
\hline $\begin{array}{l}\text { Poon et al., } \\
2013^{22}\end{array}$ & $\begin{array}{l}\text { Singapore, } \\
\text { November } \\
\text { 2001-January } 2012\end{array}$ & $\begin{array}{l}\text { Retrospective } \\
\text { cohort }\end{array}$ & $\begin{array}{l}\text { Exposed cohort: } 536 \\
\text { IVF-ICSI } \\
\text { Non-exposed cohort: } \\
\text { 16,335 spontaneous } \\
\text { pregnancies }\end{array}$ & $\begin{array}{l}\text { ART pregnancies were associated with higher risk of } \\
\text { prematurity }(\mathrm{OR}=5.95,95 \% \mathrm{Cl}=4.99-7.08) \text {, LBW } \\
(\mathrm{OR}=5.54,95 \% \mathrm{Cl}=4.37-6.13) \text { and perinatal mortality } \\
(\mathrm{OR}=4.33,95 \% \mathrm{Cl}=2.05-9.12)\end{array}$ \\
\hline $\begin{array}{l}\text { Raatikainen } \\
\text { et al., } 2012^{19}\end{array}$ & $\begin{array}{l}\text { Finland, } \\
\text { 1989-2007 }\end{array}$ & Case-control & $\begin{array}{l}\text { Cases: } 428 \text { IVF/ICSI. } \\
\text { Controls: } 18,984 \\
\text { spontaneous pregnancies }\end{array}$ & $\begin{array}{l}\text { ARTs significantly increased the risks of preterm birth } \\
(\mathrm{OR}=2.72,95 \% \mathrm{Cl}=1.02-7.22) \text { and LBW } \\
(\mathrm{OR}=1.92,95 \% \mathrm{Cl}=1.31-2.81)\end{array}$ \\
\hline $\begin{array}{l}\text { Sazonova } \\
\text { et al., 201117 }\end{array}$ & $\begin{array}{l}\text { Sweden, } \\
\text { 2002-2006 }\end{array}$ & $\begin{array}{l}\text { Retrospective } \\
\text { cohort }\end{array}$ & $\begin{array}{l}\text { Exposed cohort: } 13,544 \\
\text { IVF } \\
\text { Non-exposed cohort: } \\
587,009 \text { without ART }\end{array}$ & $\begin{array}{l}\text { IVF was associated with a higher risk of prematurity }<28 \\
\text { WOG }(\mathrm{aOR}=1.69,95 \% \mathrm{Cl}=1.34-2.14), \mathrm{LBW}<2500 \mathrm{~g} \\
(\mathrm{aOR}=1.91,95 \% \mathrm{Cl}=1.79-2.04) \text { and weight }<1500 \mathrm{~g} \\
(\mathrm{aOR}=1.72,95 \% \mathrm{Cl}=1.49-1.99) \text { in comparison with } \\
\text { spontaneous pregnancy }\end{array}$ \\
\hline $\begin{array}{l}\text { Sazonova } \\
\text { et al., 2012 }\end{array}$ & $\begin{array}{l}\text { Sweden, } \\
\text { 2002-2006 }\end{array}$ & $\begin{array}{l}\text { Retrospective } \\
\text { cohort }\end{array}$ & $\begin{array}{l}\text { Exposed cohort: } 11,292 \\
\text { IVF } \\
\text { Non-exposed cohort: } \\
571,914 \text { spontaneous } \\
\text { pregnancies }\end{array}$ & $\begin{array}{l}\text { ARTs were related to a higher rate of prematurity } \\
(\mathrm{aOR}=1.92,95 \% \mathrm{Cl}=1.12-3.93) \text {, macrosomia } \\
(\mathrm{aOR}=1.29,95 \% \mathrm{Cl}=1.04-1.59) \text { and preeclampsia } \\
(\mathrm{aOR}=1.25,95 \% \mathrm{Cl}=1.03-1.51) \text { in comparison with } \\
\text { spontaneous pregnancies }\end{array}$ \\
\hline $\begin{array}{l}\text { Sun et al., } \\
2014^{8}\end{array}$ & $\begin{array}{l}\text { China, } \\
\text { 2004-2008 }\end{array}$ & Case-control & $\begin{array}{l}\text { Cases: } 1327 \text { IVF/ICSI } \\
\text { Controls: } 5222 \\
\text { spontaneous pregnancies }\end{array}$ & $\begin{array}{l}\text { The ART group had a higher incidence of } \mathrm{C} \text {-section } \\
(\mathrm{OR}=1.40 ; 95 \% \mathrm{Cl}=1.24-1.60) \text {, prematurity and low } \\
\text { birth weight }(\mathrm{p}<0.001)\end{array}$ \\
\hline
\end{tabular}


Table 1. Characteristics of studies published in indexed journals between 2010 and 2018 addressing obstetric and perinatal complications between pregnancies resulting from IVF and ICSI and spontaneous conceptions (Continued)

\begin{tabular}{|c|c|c|c|c|}
\hline Study & Country, period & Design & Population & Reported complications \\
\hline $\begin{array}{l}\text { Sundh et al., } \\
2014^{59}\end{array}$ & $\begin{array}{l}\text { Sweden, Denmark, } \\
\text { Finland and } \\
\text { Norway, } \\
\text { 1982-2007 }\end{array}$ & $\begin{array}{l}\text { Retrospective } \\
\text { cohort }\end{array}$ & $\begin{array}{l}\text { Exposed cohort: } 91,796 \\
\text { IVF-ICSI } \\
\text { Non-exposed cohort: } \\
358,419 \text { spontaneous } \\
\text { pregnancies }\end{array}$ & $\begin{array}{l}\text { An increased risk was observed for two out of } 12 \text { types } \\
\text { of cancer in the ART group: CNS tumors, aOR }=1.44 \\
\text { and } 95 \% \mathrm{Cl}=1.01-2.05 \text { and malignant epithelial } \\
\text { neoplasms, aOR }=2.03 \text { and } 95 \% \mathrm{Cl}=1.06-3.89\end{array}$ \\
\hline $\begin{array}{l}\text { Suzuki et al., } \\
2010^{25}\end{array}$ & $\begin{array}{l}\text { Japan, } \\
2000-2007\end{array}$ & Case-control & $\begin{array}{l}\text { Cases: } 64 \text { IVF } \\
\text { Controls: } 87 \text { spontaneous } \\
\text { pregnancies. }\end{array}$ & $\begin{array}{l}\text { The C-section rate in the IVF group was higher ( } 86 \%) \\
\text { than in the control group }(67 \%) \text {, with the difference } \\
\text { being statistically significant }(p<0.01)\end{array}$ \\
\hline $\begin{array}{l}\text { Tomic et al., } \\
2011^{60}\end{array}$ & $\begin{array}{l}\text { Croatia, } \\
\text { 2006-2009 }\end{array}$ & Case-control & $\begin{array}{l}\text { Cases: } 283 \text { IVF. } \\
\text { Controls: } 283 \\
\text { spontaneous } \\
\text { pregnancies. }\end{array}$ & $\begin{array}{l}\text { Singleton pregnancies with IVF in primiparous women } \geq 35 \\
\text { years had a higher number of } C \text {-sections in comparison } \\
\text { with spontaneous pregnancies }(p<0.0001) \text { and LBW } \\
(p<0.05)\end{array}$ \\
\hline $\begin{array}{l}\text { Toshimitsu } \\
\text { et al., } 2014^{26}\end{array}$ & $\begin{array}{l}\text { Japan, } \\
\text { 2006-2010 }\end{array}$ & Case-control & $\begin{array}{l}\text { Cases: } 88 \text { IVF-ICSI } \\
\text { Controls: } 242 \\
\text { spontaneous pregnancies }\end{array}$ & $\begin{array}{l}\text { Statistically significant increase in gestational } \\
\text { hypertension with ART }(p<0.002)\end{array}$ \\
\hline $\begin{array}{l}\text { Valenzuela- } \\
\text { Alcaraz et al., } \\
2018^{33}\end{array}$ & $\begin{array}{l}\text { Spain, } \\
2014-2016\end{array}$ & $\begin{array}{l}\text { Prospective } \\
\text { cohort }\end{array}$ & $\begin{array}{l}\text { Exposed cohort: } 50 \\
\text { IVF-ICSI } \\
\text { Non-exposed cohort: } 50 \\
\text { spontaneous pregnancies }\end{array}$ & $\begin{array}{l}\text { ART twin fetuses showed significant cardiovascular } \\
\text { changes with a predominance of right heart disease } \\
(p<0.001)\end{array}$ \\
\hline $\begin{array}{l}\text { Wen et al., } \\
201028\end{array}$ & $\begin{array}{l}\text { Canada, } \\
1996-2005\end{array}$ & $\begin{array}{l}\text { Retrospective } \\
\text { cohort }\end{array}$ & $\begin{array}{l}\text { Exposed cohort: } 1044 \\
\text { IVF-ICSI } \\
\text { Non-exposed cohort: } 1910 \\
\text { spontaneous pregnancies }\end{array}$ & $\begin{array}{l}\text { ARTs were related to a higher risk of preeclampsia } \\
(\mathrm{aOR}=2.15,95 \% \mathrm{Cl}=1.33-3.46) \text { and a higher } \\
\text { number of cardiac } \mathrm{CM} \text { in comparison with spontaneous } \\
\text { pregnancies }(\mathrm{aOR}=4.58,95 \% \mathrm{Cl}=1.48-14.18)\end{array}$ \\
\hline $\begin{array}{l}\text { Wennberg } \\
\text { et al., } 2016^{12}\end{array}$ & $\begin{array}{l}\text { Sweden, Denmark, } \\
\text { Finland and } \\
\text { Norway } \\
\text { 1982-2007 }\end{array}$ & $\begin{array}{l}\text { Retrospective } \\
\text { cohort }\end{array}$ & $\begin{array}{l}\text { Exposed cohort: } 39,890 \\
\text { IVF-ICSI } \\
\text { Non-exposed cohort: } \\
245,600 \text { spontaneous } \\
\text { pregnancies }\end{array}$ & $\begin{array}{l}\text { The risk of prematurity, LBW, CM, SGA, fetal death, } \\
\text { C-section and obstetric hemorrhages was higher } \\
\text { in the ART group than in the group of spontaneous } \\
\text { pregnancies } \\
(p<0.001)\end{array}$ \\
\hline $\begin{array}{l}\text { Wisborg et al., } \\
2010^{7}\end{array}$ & $\begin{array}{l}\text { Denmark, } \\
\text { 1989-2006 }\end{array}$ & $\begin{array}{l}\text { Prospective } \\
\text { cohort }\end{array}$ & $\begin{array}{l}\text { Exposed cohort: } 742 \\
\text { IVF-ICSI } \\
\text { Non-exposed cohort: } \\
\text { 18,473 spontaneous } \\
\text { pregnancies }\end{array}$ & $\begin{array}{l}\text { Statistically significant increase in preterm birth }<37 \\
\text { WOG }(O R=1.53,95 \% \mathrm{Cl}=1.15-2.04) \text { and }<32 \text { WOG } \\
(\mathrm{OR}=2.33,95 \% \mathrm{Cl}=1.17-4.65) \text { in IVF-ICSI compared } \\
\text { to fertile women }\end{array}$ \\
\hline $\begin{array}{l}\text { Wisborg et al., } \\
2010^{30}\end{array}$ & $\begin{array}{l}\text { Denmark, } \\
\text { 1989-2006 }\end{array}$ & $\begin{array}{l}\text { Prospective } \\
\text { cohort }\end{array}$ & $\begin{array}{l}\text { Exposed cohort: } 730 \\
\text { IVF-ICSI } \\
\text { Non-exposed cohort: } \\
18,545 \text { spontaneous } \\
\text { pregnancies }\end{array}$ & $\begin{array}{l}\text { IVF-ICSI pregnancies were associated with an increased } \\
\text { risk of fetal death }(\mathrm{aOR}=4.08,95 \% \mathrm{Cl}=2.11-7.93)\end{array}$ \\
\hline $\begin{array}{l}\text { Yang et al., } \\
2014^{10}\end{array}$ & $\begin{array}{l}\text { China, } \\
\text { January-December } \\
2011\end{array}$ & Case-control & $\begin{array}{l}\text { Cases: } 1139 \text { IVF-ICSI } \\
\text { Controls: } 111,264 \\
\text { spontaneous } \\
\text { pregnancies }\end{array}$ & $\begin{array}{l}\text { The IVF-ICSI pregnancy group had a higher incidence } \\
\text { of } \mathrm{HBP}(\mathrm{OR}=1.27 .95 \% \mathrm{Cl}=1.04-1.60) \text {, premature } \\
\text { delivery }(\mathrm{OR}=4.53,95 \% \mathrm{Cl}=3.91-5.25) \text {, gestational } \\
\text { diabetes }(\mathrm{OR}=3.05,95 \% \mathrm{Cl}=2.57-3.60) \text { and placenta } \\
\text { previa }(\mathrm{OR}=2.18,95 \% \mathrm{Cl}=1.62-2.94)\end{array}$ \\
\hline $\begin{array}{l}\text { Yu HT et al., } \\
2018^{13}\end{array}$ & $\begin{array}{l}\text { China, } \\
\text { 2005-2016 }\end{array}$ & $\begin{array}{l}\text { Retrospective } \\
\text { cohort }\end{array}$ & $\begin{array}{l}\text { Exposed cohort: } 6372 \\
\text { IVF-ICSI } \\
\text { Non-exposed cohort: } \\
2,182,179 \text { spontaneous } \\
\text { pregnancies }\end{array}$ & $\begin{array}{l}\text { High risk of prematurity }(p=0.025), \text { LBW }<2,500 \mathrm{~g} \text { and } \\
<1,500 \mathrm{~g}(\mathrm{p}<0.001) \text { in the ART group }\end{array}$ \\
\hline $\begin{array}{l}\text { Zhu et al., } \\
2016^{24}\end{array}$ & $\begin{array}{l}\text { China, } \\
\text { 2006-2014 }\end{array}$ & $\begin{array}{l}\text { Retrospective } \\
\text { cohort }\end{array}$ & $\begin{array}{l}\text { Exposed cohort: } 2641 \\
\text { IVF-ICSI } \\
\text { Non-exposed } \\
\text { cohort:5282 } \\
\text { spontaneous } \\
\text { pregnancies }\end{array}$ & $\begin{array}{l}\text { ART pregnancies were associated with a } \\
\text { higher risk of gestational diabetes (OR = 1.99, } \\
95 \% \mathrm{Cl}=1.69-2.36) \text {, gestational hypertension } \\
(\mathrm{aOR}=2.58,95 \% \mathrm{Cl}=2.11-3.15) \text {, placenta previa } \\
(\mathrm{OR}=2.23, \mathrm{Cl} 95 \%=1.79-2.78) \text {, postpartum } \\
\text { hemorrhage }(\mathrm{OR}=2.72,95 \% \mathrm{Cl}=2.18-3.41) \text { and } \\
\text { placental adhesions }(\mathrm{OR}=2.37,95 \% \mathrm{Cl}=1.90-2.95) \text {. }\end{array}$ \\
\hline
\end{tabular}


achieved with ART. The difference was significant in seven studies. ${ }^{11,13,21,27,28,31,33-37}$

- Cesarean section: Twenty-three articles, where 47,482 conceptions by IVF-ICSI were recorded, reported elective or emergency C-section, which accounted for $52 \%$ of the complications. In 14 studies, the indication for $\mathrm{C}$-section was significant in pregnancies resulting from ART when compared with spontaneous pregnancies. ${ }^{8-10,12,15,19,22,23,25,33,34,38-40}$

- Premature and preterm rupture of membranes: Premature rupture of membranes (PROM) is defined as the rupture of membranes prior to the start of labor; it is called preterm when it occurs before 37 weeks of gestation. ${ }^{41}$ Nine articles (24 $\%$ of total) informed on PROM and preterm PROM (1322 ART conceptions), three indicated significant results in comparison with spontaneous pregnancies. ${ }^{17,23,24}$

- Obstetric hemorrhage: obstetric hemorrhage was regarded as bleeding due to placenta previa or premature placental abruption, a complication recorded in 13 articles ( $35 \%$ of total), which included 2110 pregnancies resulting from IVF-ICSI. In five studies, the results were significant when compared with those of spontaneous pregnancies. . $^{9,12,22,24,42}$

- Gestational diabetes: it was reported in 17 articles (46\% of total), which included 1455 IVF-ICSI pregnancies; five reported significant results in the comparison with spontaneous pregnancies. ${ }^{910,22,24,31}$

- Hypertensive disorders of pregnancy: these conditions included gestational hypertension, preeclampsia and eclampsia, and were recorded in 19 studies ( $51 \%$ of total), which included 8416 IVF-ICSI; these disorders were one of the most common complications. Only 11 articles indicated significant risk in comparison with spontaneous pregnancies. . $^{9,10,12,22,24,26,28,31,33,40,43}$

\section{Discussion}

Complications in IVF multiple gestations have been suggested to be similar to those occurring in spontaneous conceptions; however, IVF singleton pregnancies are associated with a higher incidence of complications. ${ }^{44,45}$ The exact reasons for this increase are not clear, but potential factors include maternal and paternal characteristics, underlying medical conditions associated with subfertility and infertility, sperm factors, use of fertility drugs, laboratory conditions during embryo culture, culture medium, cryopreservation and thawing, prenatal genetic diagnosis (if performed), differences in obstetric management or a combination of the above factors. ${ }^{46}$

In the presented research, 26 cohort and 11 case-control articles were analyzed, which included 10,717,574 births; 351,217 pregnancies resulting from ART and $10,366,357$ spontaneous pregnancies.

The most common perinatal complications were prematurity and low birth weight. The most important risk factor for preterm birth has been referred to be multiple gestation, which originates in a higher proportion in ART pregnancies. In addition, a higher incidence of premature births has been identified in ART singleton deliveries in comparison with children conceived in single spontaneous pregnancies. ${ }^{47}$ As for maternal complications, a high proportion of $\mathrm{C}$-section indications and hypertensive disorders of pregnancy was detected; the former might be related to anxiety and decision of the doctor given the conditions of the pregnancy. ${ }^{48}$

Elective C-section increases morbidity and mortality in comparison with vaginal delivery ${ }^{49}$ and represents a risk of maternal-fetal complication, since it is associated with hemorrhage, infections, injury to adjacent organs and venous thromboembolism, among other maternal consequences. ${ }^{50}$ Transient tachypnea, respiratory distress and immune response impairment can occur in neonates. ${ }^{51}$

Although gestational diabetes is significantly associated with factors such as obesity, ${ }^{52}$ body mass index $>30,{ }^{53}$ age $>35$ years, insulin resistance and elevated triglyceride levels, ${ }^{54}$ five articles reported that the risk of experiencing it was significantly higher in ART pregnancies, in comparison with spontaneous conceptions.

Congenital cardiovascular malformations were the most common malformations, similar to that which was reported in another research. ${ }^{55}$

One strength of our work was the period that was examined (almost one decade), the selection of articles that considered spontaneous pregnancies and the different sources of the articles.

IVF and ICSI have been found to increase the described complications mainly in European and United States populations; however, further research is required on the subject in Latin America.

In 2012, Pandey et al. ${ }^{44}$ reported similar results to those of the present work, which shows that adverse effects persist despite technological advances in ART. In a meta-analysis of ART singleton pregnancies compared to spontaneous pregnancies, Qin et $a .^{56}$ identified a higher risk of hypertensive disorders of pregnancy, gestational diabetes, C-section, prematurity and very low birth weight. On the other hand, 
Roque et al. ${ }^{57}$ reported only some maternal complications (preeclampsia, placenta accreta) when comparing ART with fresh or thawed embryos.

Given that currently the Diagnóstico de la pareja infértil y tratamiento con técnicas de baja complejidad (Diagnosis of the infertile couple and treatment with low-complexity techniques) ${ }^{58}$ clinical practice guidelines do not establish the risks associated with ART, it is essential to provide more attention and care to pregnancies resulting from ART.

\section{Conclusions}

Different factors during a spontaneous pregnancy can result in obstetric and perinatal complications, to which other factors are added in pregnancies achieved by ARTs such as IVF or ICSI, 59,60 which increase the risk of experiencing said complications. Couples who decide and are candidates for ART should receive complete and clear information that includes the risk factors and complications of the procedure they are to undergo, in addition to careful preconception counselling aimed at optimizing general health, identification of reproductive disorders and at the possibility for these pregnancies to be managed as high-risk pregnancies.

Finally, more prospective cohort studies are needed in order to identify which IVF and ICSI factors influence on the development of complications and how they could be minimized or prevented.

\section{References}

1. International Committee for Monitoring Assisted Reproductive Technology, de Mouzon J, Lancaster P, Nygren KG, Sullivan E, Zegers-Hochschild F, et al. World Collaborative Report on Assisted Reproductive Technology. Hum Reprod. 2009;24:2310-2320.

2. Halliday JL, Ukoumunne OC, Baker HWG, Breheny S, Jaques AM, Garrett C, et al. Increased risk of blastogenesis birth defects, arising in the first 4 weeks of pregnancy, after assisted reproductive technologies. Hum Reprod. 2010;25:59-65.

3. Kaser DJ, Racowsky C. Clinical outcomes following selection of human preimplantation embryos with time-lapse monitoring: a systematic review. Hum Reprod Update. 2014;20:617-631.

4. Ombelet W, Peeraer K, de Sutter P, Gerris J, Bosmans E, Martens G, et al. Perinatal outcome of ICSI pregnancies compared with a matched group of natural conception pregnancies in Flanders (Belgium): a cohort study. Reprod Biomed Online. 2005;11:244-253

5. Berntsen S, Söderström-Anttila V, Wennerholm UB, Laivuori H, Loft A Oldereid NB, et al. The health of children conceived by ART: "the chicken or the egg?" Hum Reprod Update. 2019;25:137-158.

6. Källén B, Finnström O, Lindam A, Nilsson E, Nygren KG, Olausson-Otterblad $P$. Selected neonatal outcomes in dizygotic twins after IVF versus non-IVF pregnancies. BJOG. 2010;117:676-682.

7. Wisborg K, Ingerslev HJ, Henriksen TB. In vitro fertilization and preterm delivery, low birth weight, and admission to the neonatal intensive care unit: a prospective follow-up study. Fertil Steril. 2010;94:2102-2106.

8. Sun LM, Lanes A, Kingdom JCP, Cao H, Kramer M, Wen SW, et al. intrapartum interventions for singleton pregnancies arising from assisted reproductive technologies. J Obstet Gynaecol Can. 2014;36:795-802.

9. Caserta D, Bordi G, Stegagno M, Filippini F, Podagrosi M, Roselli D, et al Maternal and perinatal outcomes in spontaneous versus assisted conception twin pregnancies. Eur J Obstet Gynecol Reprod Biol. 2014;174:64-69.
10. Yang X, Li Y, Li C, Zhang W. Current overview of pregnancy complications and live-birth outcome of assisted reproductive technology in mainland China. Fertil Steril. 2014;101:385-391.

11. Pelkonen S, Hartikainen AL, Ritvanen A, Koivunen R, Martikainen $H$, Gissler M, et al. Major congenital anomalies in children born after frozen embryo transfer: a cohort study 1995-2006. Hum Reprod. 2014; 29:1552-1557.

12. Wennberg AL, Opdahl S, Bergh C Aaris-Henningsen AK, Gissler M, Romundstad LB, et al. Effect of maternal age on maternal and neonatal outcomes after assisted reproductive technology. Fertil Steril. 2016;106:1142-1149.

13. Yu HT, Yang Q, Sun XX, Chen GW, Qian NS, Cai RZ, et al. Association of birth defects with the mode of assisted reproductive technology in a Chinese data-linkage cohort. Fertil Steril. 2018;109:849-856.

14. Valenzuela-Alcaraz B, Crispi F, Manau D, Cruz-Lemini M, Borras A Balasch J, et al. Differential effect of mode of conception and infertility treatment on fetal growth and prematurity. J Matern Neonatal Med. 2016;29:3879-3884.

15. Harlev A, Walfisch A, Oran E, Har-Vardi I, Friger M, Lunenfeld E, et al. The effect of fertility treatment on adverse perinatal outcomes in women aged at least 40 years. Int J Gynecol Obstet. 2018:140:98-104.

16. Henningsen AKA, Pinborg A, Lidegaard $\varnothing$, Vestergaard C, Forman JL, Andersen AN. Perinatal outcome of singleton siblings born after assisted reproductive technology and spontaneous conception: Danish national sibling-cohort study. Fertil Steril. 2011:95:959-963.

17. Sazonova A, Källen K, Thurin-Kjellberg A, Wennerholm UB, Bergh C. Obstetric outcome after in vitro fertilization with single or double embryo transfer. Hum Reprod. 2011;26:442-450.

18. Moini A, Shiva M, Arabipoor A, Hosseini R, Chehrazi M, Sadeghi M. Obstetric and neonatal outcomes of twin pregnancies conceived by assisted reproductive technology compared with twin pregnancies conceived spontaneously: a prospective follow-up study. Eur J Obstet Gynecol Reprod Biol. 2012;165:29-32.

19. Raatikainen K, Kuivasaari-Pirinen $P$, Hippeläinen M, Heinonen S. Comparison of the pregnancy outcomes of subfertile women after infertility treatment and in naturally conceived pregnancies. Hum Reprod. 2012;27:1162-1169.

20. Farhi A, Reichman B, Boyko V, Hourvitz A, Ron-El R L-GL. Maternal and neonatal health outcomes following assisted reproduction. Reprod Biomed Online. 2013;26:454-461.

21. Farhi A, Reichman B, Boyko V, Mashiach S, Hourvitz A, Margalioth EJ, et al. Congenital malformations in infants conceived following assisted reproductive technology in comparison with spontaneously conceived infants. J Matern Neonatal Med. 2013;26:1171-1179.

22. Poon WB, Lian WB. Perinatal outcomes of intrauterine insemination clomiphene pregnancies represent an intermediate risk group compared with in vitro fertilisation/intracytoplasmic sperm injection and naturally conceived pregnancies. J Paediatr Child Health. 2013;49:733-740.

23. Bassiouny YA, Bayoumi YA, Gouda HM, Hassan AA. Is intracytoplasmic sperm injection (ICSI) associated with higher incidence of congenital anomalies? A single center prospective controlled study in Egypt. J Matern Neonatal Med. 2014;27(3):279-282.

24. Zhu L, Zhang Y, Liu Y, Zhang R, Wu Y, Huang Y, et al. Maternal and live-birth outcomes of pregnancies following assisted reproductive technology: a retrospective cohort study. Sci Rep. 2016:6:1-11.

25. Suzuki S, Miyake H. Perinatal outcomes of elderly primiparous dichorionic twin pregnancies conceived by in vitro fertilization compared with those conceived spontaneously. Arch Gynecol Obstet. 2010;281:87-90.

26. Toshimitsu M, Nagamatsu T, Nagasaka T, Iwasawa-Kawai Y, Komatsu A, Yamashita $T$, et al. Increased risk of pregnancy-induced hypertension and operative delivery after conception induced by in vitro fertilization/ intracytoplasmic sperm injection in women aged 40 years and older. Fertil Steril. 2014;102:1065-1070

27. Lerner-Geva L, Boyko V, Ehrlich S, Mashiach S, Hourvitz A, Haas J, et al. Possible risk for cancer among children born following assisted reproductive technology in Israel. Pediatr Blood Cancer. 2017;64:1-6.

28. Wen SW, Leader A, White RR, Léveillé MC, Wilkie V, Zhou J, et al. A comprehensive assessment of outcomes in pregnancies conceived by in vitro fertilization/intracytoplasmic sperm injection. Eur J Obstet Gynecol Reprod Biol. 2010;150:160-165.

29. Vasario E, Borgarello V, Bossotti C, Libanori E, Biolcati M, Arduino S, et al. IVF twins have similar obstetric and neonatal outcome as spontaneously conceived twins: a prospective follow-up study. Reprod Biomed Online. 2010;21:422-428.

30. Wisborg K, Ingerslev HJ, Henriksen TB. IVF and stillbirth: a prospective follow-up study. Hum Reprod. 2010;25:1312-1316.

31. Yang M, Fan XB, Wu JN, Wang JM. Association of assisted reproductive technology and multiple pregnancies with the risks of birth defects and stillbirth: a retrospective cohort study. Sci Rep. 2018;8:1-8.

32. Messerschmidt A, Olischar M, Birnbacher R, Weber M, Pollak A, Leitich H. Perinatal outcome of preterm infants $<1500 \mathrm{~g}$ after IVF pregnancies compared with natural conception. Arch Dis Child Fetal Neonatal Ed. 2010;95:225-230. 
33. Valenzuela-Alcaraz B, Cruz-Lemini M, Rodríguez-López M, Goncé A García-Otero L, Ayuso H, et al. Fetal cardiac remodeling in twin pregnancy conceived by assisted reproductive technology. Ultrasound Obstet Gynecol. 2018;51:94-100.

34. Panagiotopoulou O, Fouzas S, Sinopidis X, Mantagos SP, Dimitriou G Karatza AA. Congenital heart disease in twins: the contribution of type of conception and chorionicity. Int J Cardiol. 2016;218:144-149.

35. Boulet SL, Kirby RS, Reefhuis J, Zhang Y, Sunderam S, Cohen B, et al. Assisted reproductive technology and birth defects among liveborn infants in Florida, Massachusetts, and Michigan, 2000-2010. JAMA Pediatr. 2016;170:1-9.

36. Kermani RM, Farhangniya $M$, Shahzadeh-Fazeli SA, Bagheri $P$ Ashrafi M, Vosough-Taqi-Dizaj A. Congenital malformations in singleton infants conceived by assisted reproductive technologies and singleton infants by natural conception in Tehran, Iran. Int J Fertil Steril. 2018; 11:304-308.

37. Källén B, Finnström O, Lindam A, Nilsson E, Nygren KG, Otterblad PO Congenital malformations in infants born after in vitro fertilization in Sweden. Birth Defects Res A Clin Mol Teratol. 2010;88:137-143.

38. Jackson S, Hong C, Wang ET, Alexander C, Gregory KD, Pisarska MD Pregnancy outcomes in very advanced maternal age pregnancies: the impact of assisted reproductive technology. Fertil Steril. 2015 103:76-80.

39. Kosteria I, Tsangaris GT, Gkourogianni A, Anagnostopoulos A, Papadopoulou A, Papassotiriou I, et al. Proteomics of children born after intracytoplasmic sperm injection reveal indices of an adverse cardiometabolic profile. J Endocr Soc. 2017;1:288-301.

40. Sazonova A, Kllen K, Thurin-Kjellberg A, Wennerholm UB, Bergh C Obstetric outcome in singletons after in vitro fertilization with cryopreserved/thawed embryos. Hum Reprod. 2012;27:1343-1350.

41. Committee on Practice Bulletins-Obstetrics. ACOG practice bulletin no. 188: prelabor rupture of membranes. Obstet Gynecol. 2018:131:e1-e14.

42. Healy DL, Breheny S, Halliday J, Jaques A, Rushford D, Garrett C, et al. Prevalence and risk factors for obstetric haemorrhage in 6730 singleton births after assisted reproductive technology in Victoria Australia. Hum Reprod. 2010;25:265-274.

43. Opdahl S, Henningsen AA, Tiitinen A, Bergh C, Pinborg A, Romundstad PR, et al. Risk of hypertensive disorders in pregnancies following assisted reproductive technology: a cohort study from the CoNARTaS group. Hum Reprod. 2015:30:1724-1731.

44. Pandey S, Shetty A, Hamilton M, Bhattacharya S, Maheshwari A. Obstetric and perinatal outcomes in singleton pregnancies resulting from IVF/ ICSI: a systematic review and meta-analysis. Hum Reprod Update. 2012;18:485-503

45. Schieve LA, Meikle SF, Ferre C, Peterson HB, Jeng G, Wilcox LS. Low and very low birth weight in infants conceived with use of assisted reproductive technology. N Engl J Med. 2002;346:731-737.
46. Paulson R. Pregnancy outcome after assisted reproductive technology. UpToDate [sitio web]; 2019.

47. Sanchis-Calvo A, Marcos-Puig B, Juan-García L, Morales-Suárez-Varela MM, Abeledo-Gómez A, Balanzá-MacHancosa R, et al. Características de los recién nacidos tras fecundación in vitro. An Pediatr. 2009;70:333-339.

48. Talaulikar VS, Arulkumaran S. Maternal, perinatal and long-term outcomes after assisted reproductive techniques (ART): implications for clinical practice. Eur J Obstet Gynecol Reprod Biol. 2013;170:13-19.

49. Sandall J, Tribe RM, Avery L, Mola G, Visser GH, Homer CS, et al. Short-term and long-term effects of caesarean section on the health of women and children. Lancet. 2018;392:1349-1357.

50. Burke $\mathrm{C}$, Allen R. Complications of cesarean birth: clinical recommendations for prevention and management. MCN Am J Matern Child Nurs. 2019;1-7.

51. Blustein J, Liu J. Time to consider the risks of caesarean delivery for long term child health. BMJ. 2015;350:1-4.

52. Doherty DA, Magann EF, Francis J, Morrison JC, Newnham JP. Pre-pregnancy body mass index and pregnancy outcomes. Int J Gynecol Obstet. 2006:95:242-247.

53. Torloni MR, Betrán AP, Horta BL, Nakamura MU, Atallah AN, Moron AF, et al. Prepregnancy BMI and the risk of gestational diabetes: A systematic review of the literature with meta-analysis. Obes Rev. 2009;10:194-203.

54. Yen IW, Lee CN, Lin MW, Fan KC, Wei JN, Chen KY, et al. Overweight and obesity are associated with clustering of metabolic risk factors in early pregnancy and the risk of GDM. PLoS One. 2019;14:e0225978.

55. Fedder J, Loft A, Parner ET, Rasmussen S, Pinborg A. Neonatal outcome and congenital malformations in children born after ICSI with testicular or epididymal sperm: a controlled national cohort study. Hum Reprod. 2013;28:230-240.

56. Qin JB, Sheng XQ, Wang H, Chen GC, Yang J, Yu H, et al. Worldwide prevalence of adverse pregnancy outcomes associated with in vitro fertilization/intracytoplasmic sperm injection among multiple births: a systematic review and meta-analysis based on cohort studies. Arch Gynecol Obstet. 2017;295:577-597.

57. Roque M, Valle M, Sampaio M, Geber S. Obstetric outcomes after fresh versus frozen-thawed embryo transfers: a systematic review and meta-analysis. JBRA Assist Reprod. 2018;22:253-260.

58. Instituto Mexicano del Seguro Social.Diagnóstico de la pareja infértil y tratamiento con técnicas de baja complejidad. Guía de práctica clínica. México: IMSS; 2012.

59. Sundh KJ, Henningsen AKA, Källen K, Bergh C, Romundstad LB, Gissler $\mathrm{M}$, et al. Cancer in children and young adults born after assisted reproductive technology: a Nordic cohort study from the Committee of Nordic ART and Safety (CoNARTaS). Hum Reprod. 2014;29:2050-2057.

60. Tomic V, Tomic J. Neonatal outcome of IVF singletons versus naturally conceived in women aged 35 years and over. Arch Gynecol Obstet. 2011:284:1411-1416. 